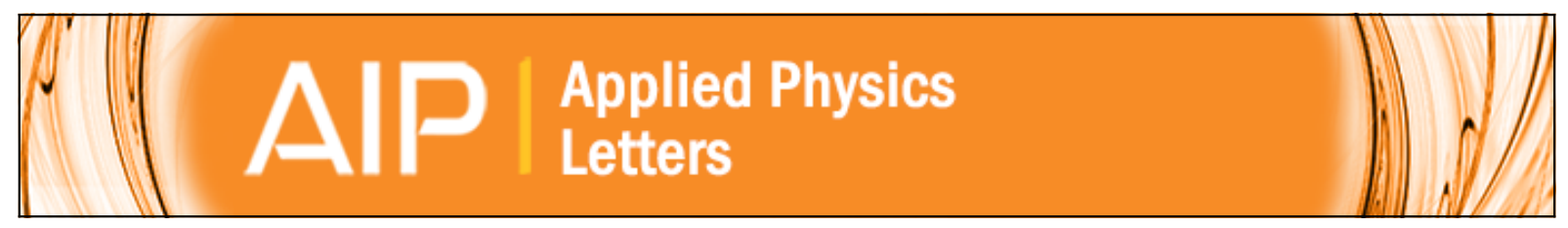

\title{
Effects of excitation density on cathodoluminescence from GaN
}

S. O. Kucheyev, M. Toth, M. R. Phillips, J. S. Williams, and C. Jagadish

Citation: Applied Physics Letters 79, 2154 (2001); doi: 10.1063/1.1408273

View online: http://dx.doi.org/10.1063/1.1408273

View Table of Contents: http://scitation.aip.org/content/aip/journal/apl/79/14?ver=pdfcov

Published by the AIP Publishing

\section{Articles you may be interested in}

Time-resolved cathodoluminescence and photocurrent study of the yellow band in Si-doped GaN

J. Appl. Phys. 94, 2341 (2003); 10.1063/1.1592296

Cathodoluminescence of epitaxial GaN laterally overgrown on (0001) sapphire substrate. Time evolution with low energy electron beam

J. Appl. Phys. 89, 7966 (2001); 10.1063/1.1373703

Luminescence spectroscopy of $\mathrm{GaN}$ in the high-temperature regime from room temperature to $900^{\circ} \mathrm{C}$ Appl. Phys. Lett. 77, 699 (2000); 10.1063/1.127090

Effect of reactive ion etching on the yellow luminescence of GaN

Appl. Phys. Lett. 75, 3710 (1999); 10.1063/1.125437

Depth-resolved electron-excited nanoscale-luminescence spectroscopy studies of defects near $\mathrm{GaN} / \mathrm{InGaN} / \mathrm{GaN}$ quantum wells

J. Vac. Sci. Technol. B 17, 2545 (1999); 10.1116/1.591125

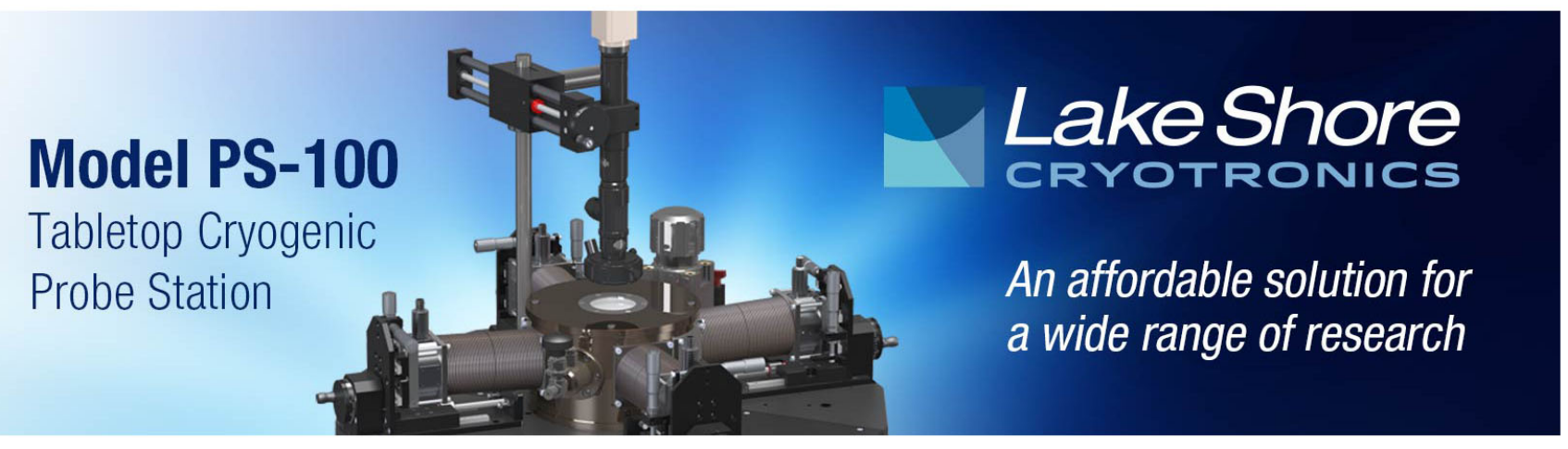




\title{
Effects of excitation density on cathodoluminescence from GaN
}

\author{
S. O. Kucheyeva) \\ Department of Electronic Materials Engineering, Research School of Physical Sciences and Engineering, \\ The Australian National University, Canberra, ACT 0200, Australia \\ M. Toth ${ }^{\text {b) }}$ and M. R. Phillips \\ Microstructural Analysis Unit, University of Technology, Sydney, Broadway, NSW 2007, Australia
}

J. S. Williams and C. Jagadish

Department of Electronic Materials Engineering, Research School of Physical Sciences and Engineering, The Australian National University, Canberra, ACT 0200, Australia

(Received 27 October 2000; accepted for publication 31 July 2001)

\begin{abstract}
Wurtzite GaN epilayers are studied by cathodoluminescence (CL) spectroscopy. Results show that the intensities of donor-acceptor pair (DAP) and yellow luminescence (YL) peaks sublinearly depend on excitation density, presumably, due to saturation effects. The intensity of near-gap emission, however, exhibits a superlinear dependence on electron-beam excitation. In contrast to photoluminescence measurements, CL studies of $\mathrm{GaN}$ are usually performed in a regime with a strongly nonlinear dependence of luminescence intensities on excitation due to a large difference in carrier generation rates for these two techniques. As a result, the ratios of near-gap to YL and DAP emission intensities strongly depend on electron-beam current. Moreover, electron-beam spot size (i.e., beam focusing) dramatically affects CL intensity. An understanding of such saturation effects is necessary for a correct interpretation of CL spectra from GaN. (C) 2001 American Institute of Physics. [DOI: 10.1063/1.1408273]
\end{abstract}

Cathodoluminescence $(\mathrm{CL})$ is a very powerful technique often used to assess the optical properties of semiconductors. ${ }^{1}$ This technique has been extensively applied to study GaN, a material of significant technological importance. ${ }^{2}$ For example, the ratios of near-gap to defectrelated emission intensities are often used as a characteristic of the quality of GaN. ${ }^{2}$ Two common defect-related CL peaks in $\mathrm{GaN}$ are (i) a ubiquitous broad defect- and/or impurity-related peak centered on $\sim 2.15 \mathrm{eV}$ at $300 \mathrm{~K}$ [often termed yellow luminescence (YL)] and (ii) donor-acceptor pair (DAP) emission (centered on $\sim 3.28 \mathrm{eV}$ at $77 \mathrm{~K}$ ), which is often observed in $\mathrm{GaN}$ at low temperatures. ${ }^{2}$ In this letter, we show that both YL and DAP emission peaks in GaN exhibit strong saturation effects which lead to a sublinear dependence of the CL intensities on excitation density. As a result, the ratios of near-gap to YL and DAP emission intensities, as well as the absolute values of CL intensities, strongly depend on both electron-beam current and beam focusing. Understanding these saturation effects is vital for a correct interpretation of CL spectra from GaN.

The samples used in this study were cut from four wafers of $\sim 2-\mu \mathrm{m}$-thick nominally undoped wurtzite $\mathrm{GaN}$ epilayers grown on $c$-plane sapphire substrates by metalorganic chemical-vapor deposition in four different EMCORE reactors. Qualitatively, the results discussed in this letter are representative of all the samples studied.

CL measurements were performed at 77 and $300 \mathrm{~K}$ using an Oxford Instruments MonoCL2 system installed on a Jeol

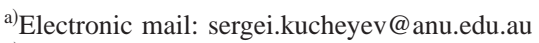

${ }^{b}$ Present address: Polymers and Colloids Group, Cavendish Laboratory, University of Cambridge, Madingley Rd, Cambridge, CB3 0HE, United Kingdom.
}

35C scanning electron microscope (SEM). CL spectra were corrected for system response. During the acquisition of CL spectra discussed here, the electron beam was scanned over a frame of $\sim 80 \times 100 \mu \mathrm{m}^{2}$ at a rate of 10 frames/s. Excitation measurements were performed by two methods: (i) changing electron-beam current while maintaining the other CL parameters constant including a finely focused beam ${ }^{3,4}$ and (ii) defocusing the electron beam by changing the strength of the SEM objective lenses, which changes beam spot size without changing beam current. The electron-beam current was measured using a Faraday cup.

Figure 1 shows CL spectra of GaN taken at $300 \mathrm{~K}$ with several values of electron-beam current. It is seen that, with decreasing beam current (i.e., excitation density), the intensities of near-gap emission (centered on $\sim 3.39 \mathrm{eV}$ ) and blue

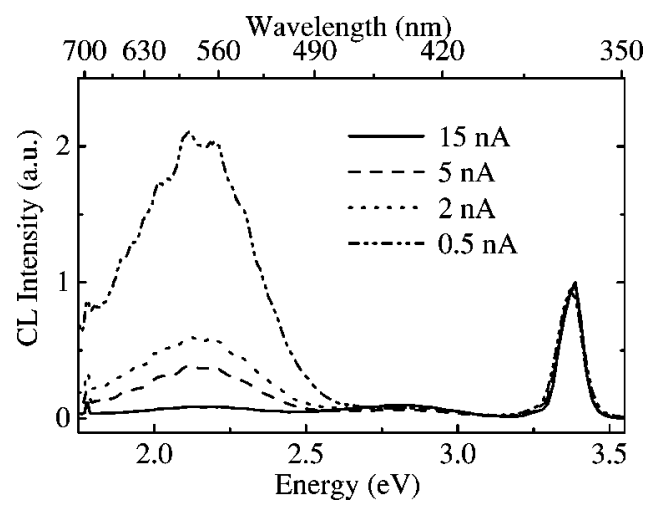

FIG. 1. CL spectra obtained from $\mathrm{GaN}$ at $300 \mathrm{~K}$ with different values of beam current (as indicated in the legend) and a finely focused electron beam (electron-beam energy $=20 \mathrm{keV}, C L$ bandpass $=2.5 \mathrm{~nm}$, scan size $\approx 80$ $\times 100 \mu \mathrm{m}^{2}$ ). The spectra were normalized to the intensities of near-gap emission peaks. 


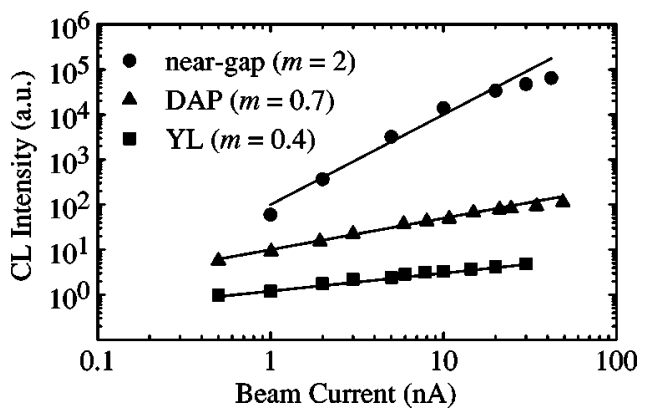

FIG. 2. Intensities of near-gap (3.45 eV), DAP (3.28 eV), and YL (2.21 eV) peaks measured at $77 \mathrm{~K}$ as a function of electron-beam current obtained with a finely focused electron beam (electron-beam energy $=20 \mathrm{keV}, \mathrm{CL}$ bandpass $=1.25 \mathrm{~nm}$, scan size $\left.\approx 80 \times 100 \mu \mathrm{m}^{2}\right)$. Power-law fits are shown as straight lines with the values of exponents $(m)$ indicated.

luminescence (a broad peak centered on $\sim 2.82 \mathrm{eV}$ ) decrease much faster than the intensity of YL (centered on $\sim 2.15 \mathrm{eV}$ ). This results in a domination of the YL band over the other peaks for relatively low values of beam current. This behavior is illustrated more fully in Fig. 2, which shows the intensities of near-gap, DAP, and YL measured at $77 \mathrm{~K}$ as a function of beam current. ${ }^{5}$ Power-law fits shown in Fig. 2 by straight lines reveal that the intensities of $\mathrm{YL}\left(I_{\mathrm{CL}}^{\mathrm{YL}}\right)$ and DAP $\left(I_{\mathrm{CL}}^{\mathrm{DAP}}\right)$ emission peaks exhibit a strongly sublinear dependence on beam current $\left(J_{b}\right)$, with $I_{\mathrm{CL}}^{\mathrm{YL}} \propto J_{b}^{0.4}$ and $I_{\mathrm{CL}}^{\mathrm{DAP}} \propto J_{b}^{0.7}$. However, the dependence of near-gap emission $\left(I_{\mathrm{CL}}^{\mathrm{NG}}\right)$ on beam current is superlinear $\left(I_{\mathrm{CL}}^{\mathrm{NG}} \propto J_{b}^{2}\right)$. ${ }^{6}$

The sublinear dependence of the YL intensity on excitation density shown above (see Figs. 1 and 2) is consistent with photoluminescence (PL) data previously reported by Grieshaber et al., ${ }^{7}$ who have shown that the intensity of YL increases close to linearly for excitation densities up to $\sim 10^{-2} \mathrm{~W} / \mathrm{cm}^{2}$, with a $\mathrm{HeCd}$ laser used as an excitation source. For higher excitation densities (up to $50 \mathrm{~W} / \mathrm{cm}^{2}$ ), they observed a strongly sublinear behavior of the YL intensity, which has been attributed to the saturation of the population of the deep level involved in YL. ${ }^{7}$ The superlinear dependence of the near-gap emission intensity on excitation density shown in Fig. 2 is also consistent with PL data reported by Müllhäuser et al. ${ }^{8}$ Such a nonlinearity of CL emission on excitation density is not unexpected since recombination via various radiative and nonradiative channels depends on excitation density. However, identification of the dominant recombination channels and their dependence on excitation density is not possible without further detailed studies.

It is worth noting that the sublinear behavior of $\mathrm{YL}$ is much less pronounced in typical PL measurements of GaN with a $\mathrm{HeCd}$ laser as an excitation source and excitation power densities typically in the range from $10^{-4}$ to $1 \mathrm{~W} / \mathrm{cm}^{2}$. Indeed, due to the fact that electron-beam excitation usually produces orders-of-magnitude greater carrier generation rates than typical optical excitation, ${ }^{1} \mathrm{CL}$ measurements of $\mathrm{GaN}$ are usually performed in a regime with a strongly nonlinear dependence of CL intensities on excitation. Hence, it is particularly important to take such a nonlinearity into account for the correct interpretation of $\mathrm{CL}$ spectra from $\mathrm{GaN}$.

In contrast to photoluminescence studies of $\mathrm{GaN}$, the strongly nonlinear dependence of $\mathrm{CL}$ intensities on excitation

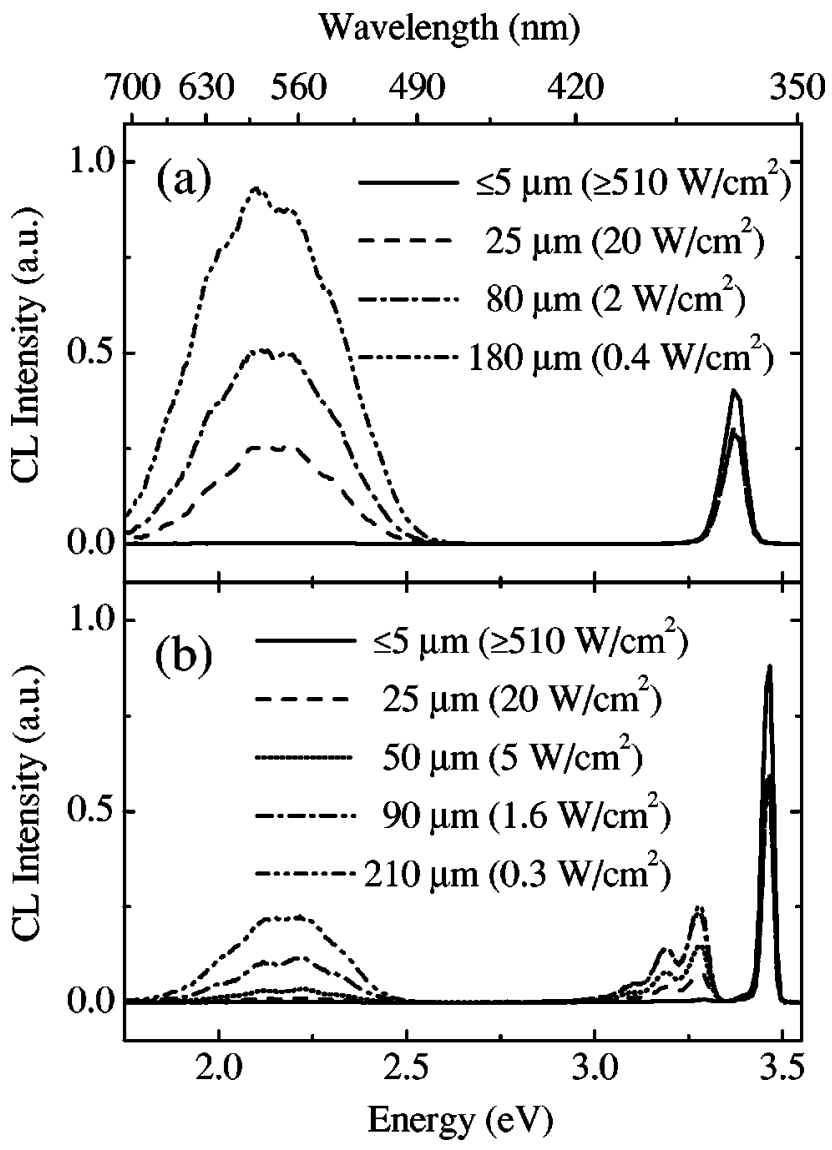

FIG. 3. CL spectra obtained from a GaN sample at $300 \mathrm{~K}$ (a) and $77 \mathrm{~K}$ (b) under different electron-beam focusing conditions [electron-beam energy $=20 \mathrm{keV}$, beam current $=5 \mathrm{nA}, \mathrm{CL}$ bandpass $=2.5$ for $(\mathrm{a})$ and $1.25 \mathrm{~nm}$ for (b), scan size $\left.\approx 80 \times 100 \mu \mathrm{m}^{2}\right]$. Beam spot diameters and corresponding instantaneous electron-beam power densities are indicated in the legend.

density discussed above significantly complicates the interpretation of $\mathrm{CL}$ data from GaN. Indeed, excitation density depends not only on the more easily controllable CL parameters (such as beam energy and current) but also on beam spot size (i.e., on the focusing of the electron beam), which is difficult to measure in a quantitative manner. This problem is illustrated in Fig. 3, which shows typical CL spectra of GaN taken at $300 \mathrm{~K}$ [Fig. 3(a)] and $77 \mathrm{~K}$ [Fig. 3(b)] with different focusing of the electron beam. We used the surface contamination produced by prolonged electron-beam bombardment ${ }^{4}$ to estimate beam spot size by subsequent secondary electron imaging of the area irradiated in spot mode. ${ }^{9}$ It is seen from Fig. 3 that, with increasing beam spot size, the intensities of YL (at both 77 and $300 \mathrm{~K}$ ) and DAP peaks exhibit a dramatic increase, accompanied by a slight decrease in the intensity of near-gap emission. This strong dependence of CL intensities on beam focusing is a direct consequence of their nonlinear dependence on excitation density. Therefore, beam spot size (i.e., beam focusing) is an important parameter (although not easily controllable in a quantitative manner) for CL measurements of GaN.

It should be noted that the decrease in the intensity of near-gap emission with increasing beam spot size (see Fig. 3) may be directly attributed to the superlinear behavior of near-gap emission on excitation density (see Fig. 2). Alternatively, a possible increase in the contribution from nonradiative surface recombination (if this process exhibits a sub- 
linear dependence on excitation) may also strongly affect the intensity of near-gap emission. Indeed, the near-gap emission detected originates mostly from a thin near-surface layer $(\sim 0.5 \mu \mathrm{m}$ thick) due to very efficient self-absorption in $\mathrm{GaN}$ (see, for example, Ref. 10). However, at present, additional work is necessary for a deeper understanding of saturation effects in $\mathrm{CL}$ from GaN. In particular, an insight into the physical processes responsible for the saturation effects in GaN may be provided by systematic studies of the influence of experimental parameters (such as electron-beam energy, sample properties, sample temperature, etc.) on CL excitation density effects. Further information may be obtained from time-resolved CL measurements of GaN.

Finally, it should be noted that a nonlinear dependence of CL intensities on excitation is not unexpected and has previously been observed in some other semiconductors (see, for example, Ref. 1, and references therein). However, relatively detailed studies of CL saturation effects have been performed only in GaAs and GaP. ${ }^{1-13}$ In these studies, ${ }^{11-13}$ a similar scale of saturation effects [i.e., similar values of exponents $(m)$ in power-law fits such as shown in Fig. 2] has been found, as compared to results of the present work on $\mathrm{GaN}$. It should be noted that, even for these relatively mature semiconductors ( $\mathrm{GaAs}$ and $\mathrm{GaP}$ ), a full understanding of $\mathrm{CL}$ saturation effects is still a challenge. However, in addition to improving our understanding of the saturation effects, a knowledge of the scale of these effects is critical for a correct interpretation of CL spectra.

In conclusion, we have shown that the ratios of near-gap to YL and DAP emission intensities, as well as the absolute values of CL intensities, strongly depend on both electronbeam current and beam focusing. Therefore, both these parameters should be taken into account for a correct interpretation of CL spectra from GaN. Finally, it should be noted that a strong nonlinearity of emission intensities in $\mathrm{GaN}$ on excitation density is much more pronounced in CL measurements than in typical PL studies due to (i) a large difference in carrier generation rates for these two techniques and (ii) a strong dependence of excitation density on the focusing of a highly localized electron beam in the case of CL.

The authors would like to thank G. Li, S. J. Pearton, and J. C. Zolper for supplying some of the samples investigated in this study.

${ }^{1}$ B. G. Yacobi and D. B. Holt, Cathodoluminescence Microscopy of Inorganic Solids (Plenum, New York, 1990).

${ }^{2}$ See, for example, a review, J. W. Orton and C. T. Foxon, Rep. Prog. Phys. 61, 1 (1998).

${ }^{3}$ It should be noted that the changing of electron-beam current was done by adjusting the condenser lens strength of the SEM, which also slightly changes beam spot size (see Ref. 4). However, in this case, the changes in excitation density due to a slight variation of beam spot size are negligible compared to those due to a large variation of beam current.

${ }^{4}$ See, for example, J. I. Goldstein, D. E. Newbury, P. Echlin, D. C. Joy, A. D. Romig, Jr., C. E. Lyman, C. Fiori, and E. Lifshin, Scanning Electron Microscopy and Microanalysis (Plenum, New York, 1992).

${ }^{5}$ In Fig. 2, we show CL data taken at $77 \mathrm{~K}$ since DAP emission is observed in $\mathrm{GaN}$ only at low temperatures.

${ }^{6} \mathrm{CL}$ excitation measurements performed at $300 \mathrm{~K}$ reveal a similar dependence of the intensities of near-gap and YL emission peaks on beam current in all samples studied.

${ }^{7}$ W. Grieshaber, E. F. Schubert, I. D. Goepfert, R. F. Karlicek, Jr., M. J. Schurman, and C. Tran, J. Appl. Phys. 80, 4615 (1996).

${ }^{8}$ J. R. Müllhäuser, O. Brandt, H. Yang, and K. H. Ploog, Mater. Res. Soc. Symp. Proc. 395, 607 (1996).

${ }^{9}$ This approach is valid under the defocused beam conditions used to acquire the spectra shown in Fig. 3 since the difference between the spot diameter and the lateral extent of the area from which (high-energy) backscattered electrons leave the sample is negligible. It should also be noted that a comparison of instantaneous electron-beam power densities given in Fig. 3 (in W/ $/ \mathrm{cm}^{2}$ ) with excitation densities in the case of PL measurements is not straightforward particularly for the smallest beam spot sizes. Indeed, the three-dimensional spatial distribution of electron energy loss in the GaN matrix should be taken into account determining beam power density in such cases.

${ }^{10}$ K. Fleischer, M. Toth, M. R. Phillips, J. Zou, G. Li, and S. J. Chua, Appl. Phys. Lett. 74, 1114 (1999).

${ }^{11}$ D. B. Wittry and D. F. Kyser, J. Appl. Phys. 38, 375 (1967).

${ }^{12}$ T. S. Rao-Sahib and D. B. Wittry, J. Appl. Phys. 40, 3745 (1969).

${ }^{13}$ H. C. Casey, Jr. and J. S. Jayson, J. Appl. Phys. 42, 2774 (1971). 